\title{
Roadside-assisted Cooperative Planning using Future Path Sharing for Autonomous Driving
}

\author{
Mai Hirata*, Manabu Tsukada*, Keisuke Okumura ${ }^{\dagger}$, Yasumasa Tamura $^{\dagger}$, Hideya Ochiai*, Xavier Défago ${ }^{\dagger}$ \\ * Graduate School of Information Science and Technology, The University of Tokyo, Tokyo, Japan \\ Email: $\{$ mai, tsukada, jo2lxq\}@hongo.wide.ad.jp \\ ${ }^{\dagger}$ School of Computing, Tokyo Institute of Technology, Tokyo, Japan \\ Email: \{okumura.k, tamura.y,defago.x\}@coord.c.titech.ac.jp
}

\begin{abstract}
Cooperative intelligent transportation systems (ITS) are used by autonomous vehicles to communicate with surrounding autonomous vehicles and roadside units (RSU). Current C-ITS applications focus primarily on real-time information sharing, such as cooperative perception. In addition to realtime information sharing, self-driving cars need to coordinate their action plans to achieve higher safety and efficiency. For this reason, this study defines a vehicle's future action plan/path and designs a cooperative path-planning model at intersections using future path sharing based on the future path information of multiple vehicles. The notion is that when the RSU detects a potential conflict of vehicle paths or an acceleration opportunity according to the shared future paths, it will generate a coordinated path update that adjusts the speeds of the vehicles. We implemented the proposed method using the open-source Autoware autonomous driving software and evaluated it with the LGSVL autonomous vehicle simulator. We conducted simulation experiments with two vehicles at a blind intersection scenario, finding that each car can travel safely and more efficiently by planning a path that reflects the action plans of all vehicles involved. The time consumed by introducing the RSU is $23.0 \%$ and $28.1 \%$ shorter than that of the stand-alone autonomous driving case at the intersection.
\end{abstract}

\section{INTRODUCTION}

Autonomous driving technologies are a part of intelligent transport systems (ITS) that use information and communication technologies to exchange pedestrian, road, and vehicles data to mitigate common road and traffic problems, including accident and congestion avoidance. Consequently, they have attracted a great deal of attention in recent years. Car companies have already commercialized related functions, such as autonomous emergency braking, lane-keeping assistance, and automatic parking [1], [2]. Several organizations are developing autonomous automated driving along with opensource software packages such as Autoware [3] and Apollo [4].

Cooperative ITS is important because autonomous vehicles cannot otherwise guarantee the safety of pedestrians, vehicles, and property. The common architecture is a station infrastructure [5], [6] that was developed by the European Telecommunications Standards Institute (ETSI) and the International Organization for Standardization (ISO). According to standards, a cooperative awareness message (CAM) [7] is the fundamental safety-related vehicle-to-vehicle (V2V) process that shares real-time data about other vehicles. Vehicle-toeverything (V2X) processes are advancements that share sensors information to vehicles and roadside equipment to create a collective perception capability. In this regard, ETSI is in the process of standardizing collective perception messages (CPMs) [8], [9].

The future information consists of a list of planned positions of a vehicle over time (i.e., the planned trajectory). According to ETSI, a maneuver coordination message (MCM) exchanges planned trajectories and performs driving coordination. However, its format is still under development [10], and no standard has been issued. Maneuver coordination (MC) research has been conducted in various scenarios such as platooning, lane changing, and merge coordination [11], [12].

In many field demonstrations, infrastructure-based CPM has been demonstrated to improve the perception of automated vehicles [13], [14]. However, research on the infrastructure which supports the vehicle's better prediction and planning is still in its nascent stage. Especially it is not thoroughly investigated with the full-fledged autonomous software.

The contribution of this study is designing a roadside unit (RSU) that enhances vehicle' prediction and planing cooperatively with other vehicles to improve traffic flow at intersections. We implemented the solution in Autoware [3], open-source software for autonomous driving systems. To the best of our knowledge, this study is the first to implement cooperative planning in Autoware. The proposed method is divided into two schemes: a periodic message broadcast to exchange vehicles' future path (i.e., future path sharing) and a maneuver coordination algorithm based on a reservation table that manages the set of future paths in the RSU (i.e., cooperative planning).

The rest of this paper is structured as follows. Section II reviews related works and Section III describes our proposal. Section IV] shows our implementation based on Autoware. Section $\mathrm{V}$ evaluates the implementation using LG Corporation's Silicon Valley Lab (LGSVL) simulator [15]. Section VI concludes the paper and provides future works.

\section{RELATED WORK}

Renzler et al. [16] proposed CAM extensions to inform future path information for emergency response in the event of collisions. It was shown to reduce evasive maneuvers that would otherwise pose a danger to vehicles in adjacent lanes.

Technologies for sharing sensor information, including ITS messages, can be broadly divided into three levels: low, 
feature, and track [17]. Track-level data exchange has the advantage of not requiring large network bandwidth. Gabb et al. [18] presented a scheme for data sent from a mobile edge computing (MEC) server to a car and proposed a system that fuses data from the car's sensors with an environmental model based on infrastructure sensor data at the MEC server. Tsukada et al. [14], [19] proposed AutoC2X, a system that shares information collected by roadside equipment with an automatic vehicle using Autoware [3] and OpenC2X, opensource experimental and prototype platforms for cooperative ITS.

Existing research on the management of autonomous vehicles at intersections can be categorized into decentralized and centralized approaches. In the decentralized approach, autonomous vehicles communicate via a wireless network. Azimi et al. [20] proposed a cooperative intersection management algorithm that models the area of an intersection as a grid. Each cell in the grid is assigned a unique identifier and shares current and future locations with vehicles. Aoki et al. [21] studied how autonomous vehicles drive through dynamic intersections in ways that lead to accidents using sensor-based perception and inter-vehicle communication. The autonomous vehicles communicate with the Intersection Manager (IM) for the crossing plan for the centralized approach. Dresner et al. [22] proposed autonomous intersection management (AIM) system for coordinating the independent movements of selfdriving cars through intersections. Liu et al. [23] proposed a trajectory-planning-based version, whereas Bashiri et al. [24] proposed a platoon-based approach.

Maneuver coordination (MC) research can be divided into two categories: scenario-specific MC and general MC. In a platooning coordination study, [25] suggested a V2X protocol for handling all possible scenarios of platooning. Vehicles are coordinated with the protocol to merge two platooning sequences into one. [26] also proposed a redundant message protocol for cooperative driving that involves various vehicles, including platooning. In the proposed system [27] for smooth ramp merging, the roadside unit at the merging point detects the merging activity while searching for vehicles on the main road with the possibility of involving in a collision. Subsequently, it instructs speed adjustment to the identified vehicles. Furthermore, the cooperative maneuver protocol (CMP) is proposed [28] as a coordination message at the merge point. For coordinated lane changing, [12] suggested lane-change messaging. Various studies have employed the space-time reservation protocol (STRP) that uses messaging for reserving a particular location for a certain period. [29] used STRP for lane-change coordination and carried out field experiments to justify the technique. In [30], the method is extended to intersections, passing, and roundabouts.

[31] put forward the TransAID project that conducts research and development on more generic V2X messages. It also involved the study of cooperative automated driving and Cooperative ITS standard messages, including CAM, CPM, and MCM [32], [33]. [32] suggested a format for a more generic MCM message. It considered the transition of control

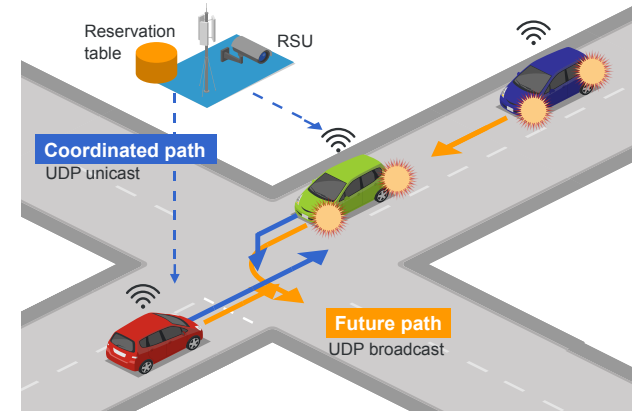

Fig. 1. Cooperative planning using future path sharing

(switching from automatic to manual driving) as an instruction type for MCM along with lane change and speed adjustment. In [33], a V2X framework was implemented for exchanging each message sets standardized in Cooperative ITS.

Furthermore, in [34], [35], a full stack from the access layer was applied to the facility layer. Besides MCM, they used a maneuver recommended message for driving coordination. For the same purpose, [36] proposed the complex vehicular interactions protocol. [37] assumed that vehicles always provide a trajectory when carrying out driving cooperation. Apart from using MCM for the reduction of uncertainty in automated vehicles, [11] proposed the MCM as a more general protocol. An Autoware-based MC protocol is implemented as AutoMCM [38], and the system demonstrates robustness against packet loss in the experiments.

We reviewed these studies and others on future path replacement, driving management at intersections, and maneuver coordination. Future path exchange methods are in their infancy, and no experimental data have yet been obtained. There have also been just a few examples of intersection driving management platforms that enable actual autonomous driving.

\section{Future Path Sharing Based Cooperative PLANNING}

Figure 1 shows an overview of our scheme. The autonomous vehicles periodically broadcast their future path information to the surrounding vehicles. Receiver vehicles make adjustments based on the perception of the other vehicles' future paths as obstacles. As a result, all vehicles that receive the future path will slow down and not pass through the intersection efficiently. In our proposal, the RSU improves the situation by collecting future paths and adding them to its reservation table. When one future path conflicts with another vehicles' future path in the reservation table, the RSU computes a conflictfree resolution (coordinated path) and sends it to the affected vehicle. When the vehicle receives a new path from the RSU, it prioritizes it over its previously prepared path. In the following sections, we describe future path sharing and coordinated path generation in detail. 


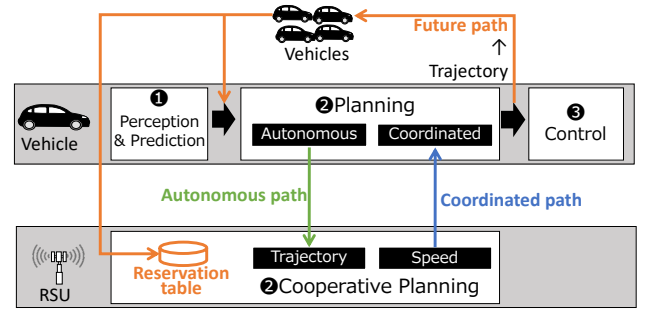

Fig. 2. Future path, autonomous path, coordinated path, and reservation table

\section{A. Future path sharing}

Figure 2 displays a typical autonomous driving system. First, the perception subsystem processes sensor inputs to interpret the surrounding environment. It detects the location, size, and type of surrounding vehicles and pedestrians and predicts their movements. The planning module then calculates a route from the map to the target point and generates an action plan along that route. As an output, the planning module generates a trajectory and provides it to the control module.

We define the following terms to describe our system:

- The trajectory consists of continuous paired sequences of the location and velocity. It results from the planning module's decision, and the control module follows the trajectory in typical autonomous driving software (e.g., Autoware).

- The future path consists of continuous paired sequences of coordinates and timing factors of their passing. The future path employs a data structure that makes it easy for cars and RSUs to detect conflicts. We generate the future path assuming that the velocity from a position to the next position is constant. The time to pass $n$-th point is calculated by equation (1).

$$
t_{n}=\sum_{k=0}^{n} \frac{x_{k}-x_{k-1}}{v_{k}}+t_{0}(n \geq 1),
$$

where $t_{0}$ is the current time, $x_{0}$ and $v_{0}$ are the pair of the location and the velocity of the closest point to the current vehicle position in the trajectory.

Our proposal converts the trajectory output from the planning module to the future path using equation (1). Then, the future path is broadcast to the surrounding vehicles with an identifier, the current position, the current speed, and the vehicle shape. The receiving vehicle can treat the received information as a dynamic obstacle by integrating it with the output of the perception module. This integration potentially causes unnecessary deceleration because the future path is treated as a future obstacle. Therefore, a coordinated path (detailed in the next section) is necessary to improve traffic flow at the intersection.

\section{B. Coordinated path generation}

We define the following terms to describe our system:

- The reservation table is the RSU's database that stores the set of future paths sent by autonomous vehicles. The reservation table is used to detect conflicts between cars and calculate opportunities for acceleration.

- An autonomous path is calculated by the autonomous vehicle to the desired destination. The vehicle periodically unicasts its autonomous path to the RSU during the coordinated mode. Expectedly, the path may conflict with other vehicles paths, causing unnecessary deceleration.

- The coordinated path is the new path in which the RSU modifies the autonomous path to avoid conflicts and improve traffic flow. The RSU periodically unicasts this information to the target vehicles, and the vehicles give priority to this path.

Our scheme is a hybrid one in which vehicles create a trajectory, and the RSU modifies their speeds along the trajectories. The RSU receives the vehicles' future paths, as shown in Fig. 2, and it updates its reservation table. When a received future path conflicts with another path in the table, the RSU sends an initiation message to a vehicle to enter a coordinated mode. As depicted in Fig. 2, the RSU then generates a coordinated path that slows the vehicle's speed until the future path is sufficiently shortened, and the conflict disappears. This could involve one vehicle ultimately stopping.

When the received future path does not conflict with any path in the reservation table, the RSU determines room for acceleration. If so, it performs the same steps, resulting in the vehicle temporarily speeding up.

\section{IMPLEMENTATION}

We implemented the proposed method by extending Autoware.IV (v.0.6.0). In this section, we describe Autoware and explain our proposed extension in detail.

\section{A. Autoware}

Autoware is a Linux-based open-source software for autonomous driving [3] and is based on the Robot Operating System (ROS) [39]. ROS is a distributed robot platform that utilizes nodes and topics. The nodes represent the processing module of a task and interact via topics. The ROS includes the RViz 3D visualization tool, which displays the status of the tasks. Autoware provides a set of applications necessary for autonomous driving, including localization, perception, planning, and control. Using 3D maps, LiDAR, a camera, and the Global Navigation Satellite System, Autoware performs localization and perception.

The perception module provides detected obstacle information to the Autoware planning module, which is divided into three parts: mission planning, scenario selection, and behavior planning. The mission planner calculates the entire route to the goal by searching a static map. The scenario selector decides which behavior planner should be applied depending on the situation. Notably, it is technically challenging to have a unified behavior planner handle every possible situation. Currently, Autoware provides two: one for on-road driving and another for parking. The selected planner gives the calculated trajectory to the control module that manipulates acceleration, braking, and steering via a controller area network. 


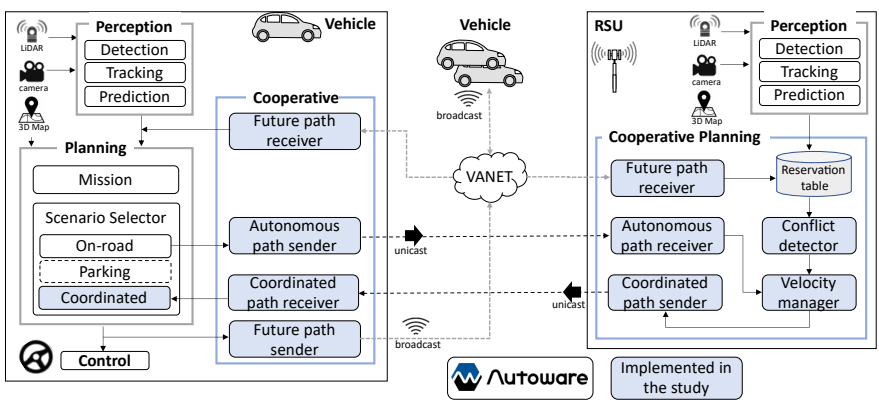

Fig. 3. Implementation overview

\section{B. Implementation overview}

Figure 3 shows the overview of our implementation. The white box comes with Autoware, and the blue box is newly implemented in our extension.

We modified the structure of ROS nodes as follows. We added the coordinated planner in addition to the on-road planner and the parking planner. The scenario selector switches between the on-road and coordinated planners depending on the mode (described later). The parking planner is not used. The planning module passes the trajectory to the control module with the topic, /planning/scenario planning/trajectory. The future-path sender converts the trajectory to a future path and sends it using a user datagram protocol (UDP) broadcast at a frequency of $10 \mathrm{~Hz}$. A future path includes 120 trajectory points, and the distance between each point should be at least $0.1 \mathrm{~m}$. The network load and the implications are analyzed in section $\mathrm{V}-\mathrm{C}$. Both vehicles and the RSU receive the future path, the future path receiver publishes it to /perception/object_recognition/objects, and the perception module sends the surrounding objects to the planning module. Hence, the planning module can also employ future data from V2X.

When the RSU receives the data and no conflicts are detected, the future path is added to the reservation table. In case of a conflict, the RSU sends an initiation message to the vehicle requesting it switch to the coordinated mode. The vehicle then activates the autonomous path sender to send its autonomous paths to the RSU periodically at a unicast of $100 \mathrm{~ms}$ intervals. Upon reception at the RSU, the velocity manager generates a coordinated path by modifying the autonomous path's speed from the autonomous path receiver. The velocity manager then decreases the vehicle's speed when a conflict is detected and increases its speed when it discovers room for acceleration. When the vehicle receives the coordinated path from the RSU, the vehicle performs planning in the coordinated mode.

As a limitation of the current implementation, it only focuses on the longitudinal vehicle motion, and the lateral vehicle motion calculation is future work. Also, our current implementation uses the JavaScript Object Notation (JSON) format [40] to send the future path, the autonomous path, and the coordinated path where the size of the data is $60 \mathrm{~KB}$ when

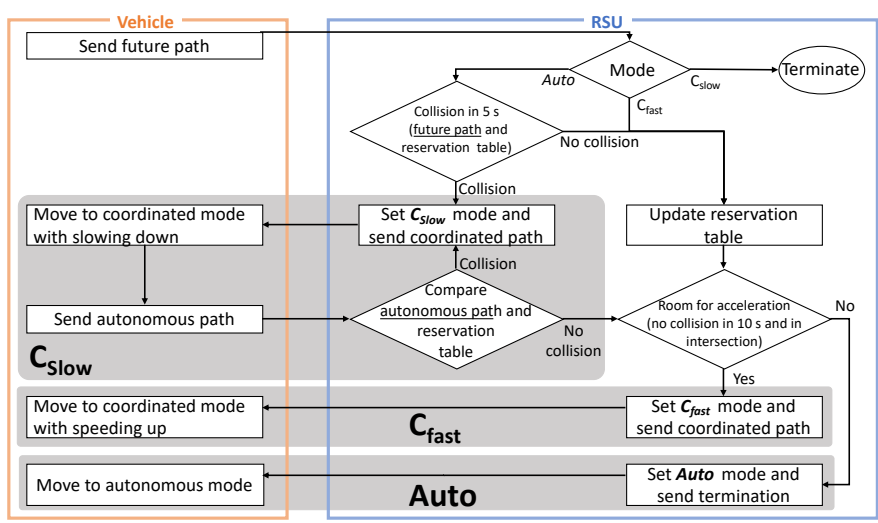

Fig. 4. Mode-switching flowchart

the path has 120 points. The future work includes reducing the packet size within the maximum transmission unit size of UDP (i.e., 1460 Bytes).

\section{Coordinated path generation with mode management}

The RSU applies one of three modes for each vehicle as follows: Auto is used when the vehicle follows the autonomous planning mode; $C_{\text {slow }}$ is used during the coordinated mode while slowing down; and $C_{\text {fast }}$ is used during the coordinated mode while speeding up. The RSU chooses the modal behavior using the process shown in Fig. 4

During the first stage, all vehicles' modes are set to Auto. The future path of the first vehicle is then added to the table. When a future path is received, the RSU checks for conflicts within $T_{\text {collision }}$ seconds with all other future paths in the reservation table. The coordinates that exist within the safety margin $D_{\text {margin }} \mathrm{m}$ are counted as conflicts. In case of no conflict, the RSU then searches for an acceleration opportunity by checking if there is also no conflict in $T_{f r e e}$ second, and the vehicle is approaching the intersection. In this implementation, we set $T_{\text {collision }}=5 \mathrm{~s}$ to allow for enough deceleration when a conflict is detected, $T_{\text {free }}=10 \mathrm{~s}$ to allow for acceleration until exiting the intersection, and $D_{\text {margin }}=2.8 \mathrm{~m}$ the width of the car.

If there is room for acceleration, the RSU changes the vehicle's mode from Auto to $C_{\text {fast }}$. To force the mode change, the RSU sends an initiation message to the vehicle to switch to $C_{\text {fast }}$. Then, the RSU and the vehicle generate a coordinated path that changes the speed of the autonomous vehicle to the maximum speed of the road $\left(V_{\max }\right)$ through the intersection as long as there is no conflict within $T_{\text {free }}$ second. The vehicle then follows the coordinated path and accelerates. Although the coordinated path's speed is faster than the vehicle's current speed, the control module of the Autoware accelerates smoothly until called off. When the vehicle passes through the intersection, the RSU changes its mode back to Auto and sends a termination message to the vehicle. Thus, the acceleration and deceleration follow a smooth curve. 
Alternatively, if the received future path conflicts with other paths in the reservation table, the RSU switches to $C_{\text {slow }}$. During this mode change, an initiation message is sent to the vehicle to switch to the $C_{\text {slow }}$ mode. After receiving the autonomous path, the RSU sends back the coordinated speed of zero. In the vehicle, Autoware's control module decelerates smoothly according to the current speed until the autonomous path no longer conflicts with the other paths in the reservation table (perhaps until stopping). During the $C_{\text {slow }}$ mode, RSU discards the vehicle's future path. When the vehicle path no longer conflicts with others, it calculates its room for acceleration and switches to one of the other modes (i.e., Auto or $C_{\text {fast }}$ ).

\section{Evaluation}

We evaluated our implementation using the LGSVL autonomous driving simulator v.2020.06 [15]. In evaluation, we verified that the RSU intervention effectively reduced the vehicle transverse time through the intersection.

\section{A. Experimental setup and scenarios}

Three personal computers (PCs) representing Car A, Car B, and the $R S U$ were connected to the LGSVL simulator. The implementations included perception, planning, and controlling within the virtual world. We chose the Shalun map (Taiwan Car Lab Testing Facility) for a blind intersection preinstalled with LGSVL. A wired network connected the three PCs to the LGSVL simulator.

The simulation experiment was conducted at a virtual intersection having poor visibility wherein two cars ( Car A and Car B) passed one another. We set the starting and destination points so that $\operatorname{Car} A$ would pass through the intersection before Car $B$ in stand-alone mode, as shown in Fig. 5). We set the maximum speed of the road $V_{\max }=50 \mathrm{~km} / \mathrm{h}$. We set a stationary vehicle as RSU in the simulator because it had full functionality apart from the control module.

We evaluated the following scenarios:

- Stand-alone: autonomous cars without communication.

- Future path only: autonomous vehicles broadcast the future path by V2V.

- Future path with RSU: coordinated autonomous driving with the RSU including future path sharing.

We conducted 10 simulation experiments for each of the three scenarios using the same starting points and destinations.

\section{B. Measurement of passing time in the intersection}

Table 1 shows all results and passing times. In the standalone case, $\operatorname{Car} A$ traversed the intersection first in all trials, as designed. On the other hand, $\operatorname{Car} A$ traversed the first six times in the future-path-only scenario and four times in the future path with RSU. Each time, the result varied because we launched the experimental setup using a graphical user interface at slightly different timing.

Table [1] shows that the future-path-only scenario slightly increased the average passing time from $36.51 \mathrm{~s}$ to $37.17 \mathrm{~s}$, which represents $2 \%$. The reason for the worse results in the

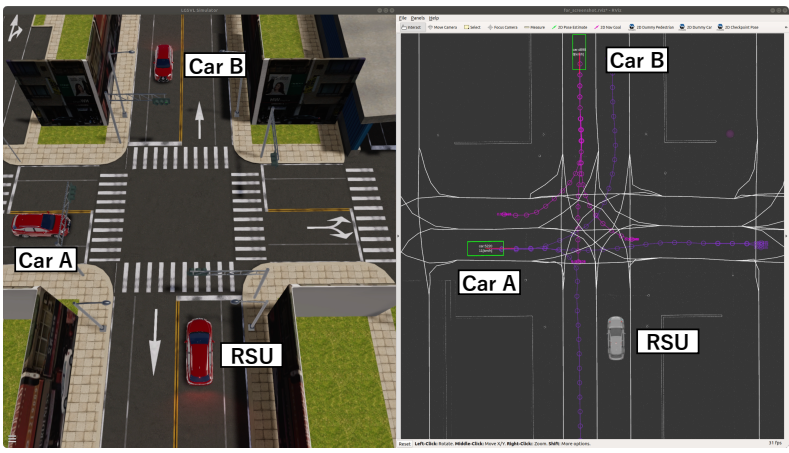

Fig. 5. Screenshot of the experiment. Car A arrives first in stand-alone mode. Left is the view of the LGSVL simulator. Right is the Rviz view.

TABLE I

PASSING TIME IN THE EXPERIMENTAL EVALUATION

\begin{tabular}{c|ccc}
\hline & Stand-alone & $\begin{array}{c}\text { Future } \\
\text { Paths only }\end{array}$ & $\begin{array}{c}\text { Future path } \\
\text { with RSU }\end{array}$ \\
\hline \hline Car A passes first & 10 & 6 & 6 \\
Car B passes first & 0 & 4 & 4 \\
\hline Car A passing time & $31.04 \mathrm{~s}$ & $38.03 \mathrm{~s}$ & $23.93 \mathrm{~s}$ \\
Car B passing time & $41.97 \mathrm{~s}$ & $36.32 \mathrm{~s}$ & $30.18 \mathrm{~s}$ \\
Average passing time & $36.51 \mathrm{~s}$ & $37.17 \mathrm{~s}$ & $27.05 \mathrm{~s}$ \\
\hline
\end{tabular}

future-path-only scenario becomes apparent later by analyzing the speed of cars passing through the intersection in Figure 7 Our proposal (the future path with RSU) successfully reduced the average passing time from $36.51 \mathrm{~s}$ to $27.05 \mathrm{~s}$, representing a $26 \%$ improvement. This result shows that the future paths of other vehicles were perceived as future obstacles that would otherwise degrade traffic flow. The coordinated path of the RSU effectively avoided conflict and improved flow.

Figure 6 shows the passing times of the 10 trials and their variations. In the stand-alone case, the results showed only a few variations because $\operatorname{Car} A$ always passed first. When the vehicles exchanged future paths, the passing times varied because $\operatorname{Car} A$ traversed the first six times and $\operatorname{Car} B$ four. As a result, $C a r A$ took $6.99 \mathrm{~s}$ longer to traverse than in the stand-alone case ( $22 \%$ slower), whereas Car B arrived $5.65 \mathrm{~s}$ earlier ( $13 \%$ faster). Our proposed method improved the passing times $23.0 \%$ and $28.1 \%$ for Car A and Car B, respectively. The proposed method reduces traversal times by $25.9 \%$ with a few variations.

Figure 7 shows the speed of the two vehicles near the intersection. Green is the result for Car A and orange for Car $B$, and the larger value indicates the direction of travel. The transparent colored line shows the speed of each trial, and the solid line shows the average speed. $(x, y)=(0,0)$ represents the intersection.

As depicted in Fig. 7(a), in the stand-alone scenario, $\operatorname{Car} B$ always stopped at $5 \mathrm{~m}$ before the intersection for $\mathrm{Car} A$ passing first. As shown in Fig. 7(b), in the future path-only scenario, Car A and Car B recognized each other. They slowed sooner (within $20 \mathrm{~m}$ from the intersection) than in the stand-alone case, which explains why the future path exchange degrades 


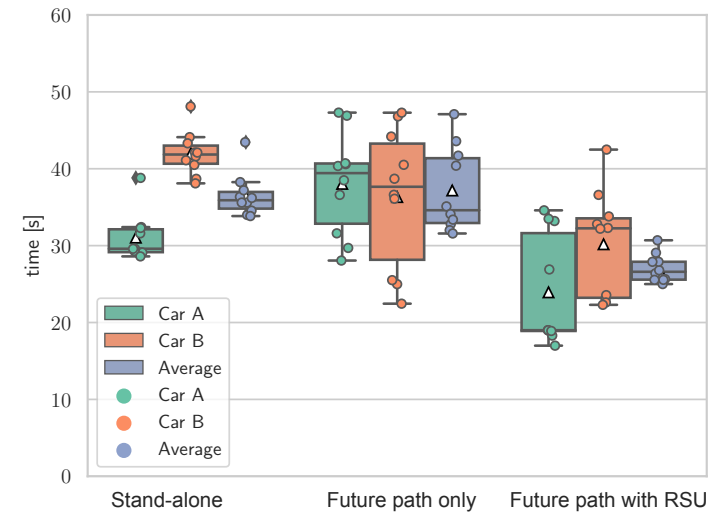

Fig. 6. Passing times of 10 results

the passing time. Figure 7(c) shows that both $\mathrm{Car} A$ and $\mathrm{Car}$ $B$ did not stop before the intersection using our proposal. The first vehicle that sent its future path to the RSU accelerated along the coordinated path, and the other vehicle decelerated sooner than in the stand-alone case.

\section{Discussion}

Unlike this experiment, actual cars would communicate via wireless networks. Therefore, the network resources are limited, and we need to consider the network load. First of all, vehicles and RSUs can send a maximum of 120 trajectory points, theoretically limited by the maximum UDP packet size (1460 Bytes), assuming that the latitude, longitude, and time are 32 bits [41]. It consumes the network bandwidth equivalent to $116 \mathrm{Kbps}$ when the maximum UDP packet is sent at $10 \mathrm{~Hz}$. In the proposed method, future paths, autonomous paths, and coordinated paths are sent at $10 \mathrm{~Hz}$, which requires $350 \mathrm{Kbps}$ of network traffic per car. The proposed method can control up to 17 cars when the data rate of wireless media is $6.0 \mathrm{Mbps}$ (i.e., DSRC). Some optimizations are necessary to coordinate more cars, such as reducing the number of trajectory points, transmission rate, etc. According to [42], fully automated, where all maneuvers are performed cooperatively, is classified as Day 4 (to be deployed by 2040-2045). Thus, the wireless media available at that time may solve the problem.

In addition, our proposal is a method for RSUs to take over the control of automobiles. It may be challenging to obtain the consent of current manufacturers of autonomous self-driving cars. Therefore, our method may be adapted in the situation where the same manager controls both cars and RSUs, such as factories.

\section{CONCLUSION AND FUTURE WORK}

In cooperative ITS, autonomous vehicles use V2X to exchange real-time information. However, real-time path planning becomes inefficient when driving paths conflict. This study proposed a method for cooperative path planning using future path sharing that considers future planning information. We placed an RSU at the intersection to coordinate path planning among two autonomous vehicles and implemented our proposition by extending Autoware, an open-source autonomous driving software, to simulate the traversal times of two vehicles at a blind intersection using the LGSVL autonomous driving simulator. The results show that future-path broadcasts improve traversal times because the autonomous vehicles adjust their speeds based on their combined future paths, governed by the RSU. Our proposal effectively coordinates vehicle speeds and achieves a $26 \%$ faster traversal time for both vehicles through the intersection.

Future work will include a large-scale simulation involving many cars and pedestrians. We used a wired network in this study. However, we plan to use a wireless network or a network simulator in the future. Furthermore, field experiments would provide a more realistic environment for evaluation. Furthermore, it is necessary to manage vehicles' priority at intersections and compare the performance with related works. The proposed scheme can be extended to mixed traffic stream non-connected cars and vulnerable road users (e.g., cyclists and pedestrians). RSU's prediction module can add the predicted paths of the dynamic objects to the reservation table and generate the conflict-free coordinated path for autonomous vehicles. The evaluation for the scenario is future work.

\section{ACKNOWLEDGMENT}

This work was partly supported by JSPS KAKENHI (grant number: 19KK0281 and 21H03423).

\section{REFERENCES}

[1] Honda, "Honda receives type designation for level 3 automated driving in japan," https://global.honda/newsroom/news/2020/4201111eng.html 11 2020, (Accessed on 02/04/2021).

[2] "Toyota bringing advanced its technology to mass-market models toyota motor corporation official global website," https://global.toyota/ en/detail/9676551 (Accessed on 02/04/2021).

[3] S. Kato, E. Takeuchi, Y. Ishiguro, Y. Ninomiya, K. Takeda, and T. Hamada, "An open approach to autonomous vehicles," IEEE Micro, vol. 35, no. 6, pp. 60-68, Nov 2015.

[4] "Apolloauto/apollo: An open autonomous driving platform," https:// github.com/ApolloAuto/apollo (Accessed on 2021/07/24).

[5] "ISO 21217:2020 Intelligent transport systems — Station and communication architecture." ISO/TC 204 Intelligent transport systems, December 2020.

[6] "Intelligent Transport Systems (ITS); Communications Architecture." ETSI EN, September 2010, ETSI EN 302665 V1.1.1 (2010-09).

[7] "ITS; Vehicular Communications; Basic Set of Applications; Part 2: Specification of Cooperative Awareness Basic Service," Dec. 2014, ETSI EN 302 637-2 V1.3.2 (2014-11)

[8] ITS; Cooperative Perception Services (CPS), ETSI Std., Dec. 2019, ETSI TS 103324 V0.0.14 (2019-10).

[9] ITS; Vehicular Communications; Basic Set of Applications; Analysis of the Collective Perception Service (CPS); Release 2 , ETSI Std., Dec. 2019, ETSI TR 103562 V2.1.1 (2019-12).

[10] ITS; Vehicular Communications; Informative report for the Maneuver Coordination Service, ETSI Std., May 2020, ETSI TR 103578 (Early draft).

[11] B. Lehmann, H. Günther, and L. Wolf, "A generic approach towards maneuver coordination for automated vehicles," in 2018 21st International Conference on Intelligent Transportation Systems (ITSC), Nov. 2018, pp. 3333-3339.

[12] L. Wang, R. F. Iida, and A. M. Wyglinski, "Coordinated lane changing using V2V communications," in 2018 IEEE 88th Vehicular Technology Conference (VTC-Fall), Aug. 2018, pp. 1-5.

[13] M. Shan, K. Narula, Y. F. Wong, S. Worrall, M. Khan, P. Alexander, and E. Nebot, "Demonstrations of cooperative perception: Safety and robustness in connected and automated vehicle operations," Sensors, vol. 21 , no. 1 , p. 200 , Dec. 2020. 


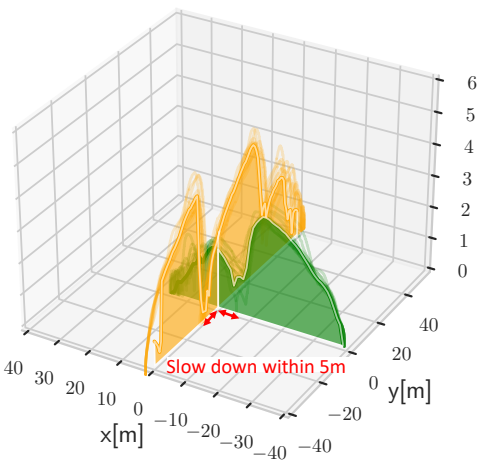

(a) Stand-alone

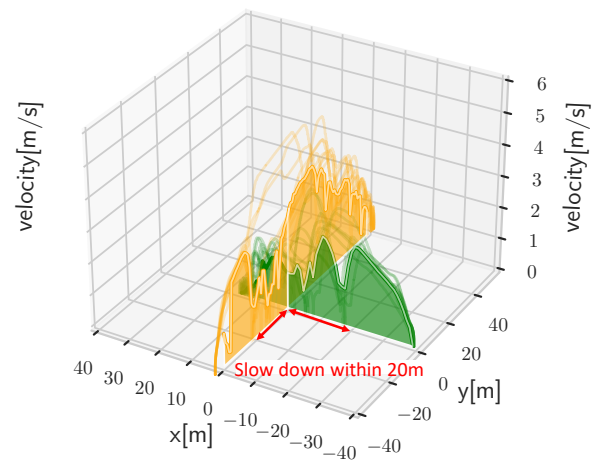

(b) Future path only

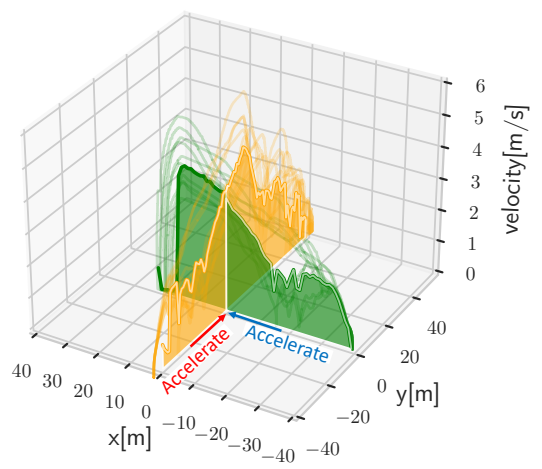

(c) Future path with RSU

Fig. 7. Speed of two vehicles near the intersection

[14] M. Tsukada, T. Oi, A. Ito, M. Hirata, and H. Esaki, "Autoc2x: Open-source software to realize $\mathrm{v} 2 \mathrm{x}$ cooperative perception among autonomous vehicles," in The 2020 IEEE 92nd Vehicular Technology Conference (VTC2020-Fall), Victoria, B.C., Canada, 2020.

[15] G. Rong et al., "LGSVL simulator: A high fidelity simulator for autonomous driving," in 2020 IEEE 23rd International Conference on Intelligent Transportation Systems (ITSC), Sep. 2020, pp. 1-6.

[16] T. Renzler, M. Stolz, and D. Watzenig, "Looking into the path future: Extending cams for cooperative event handling," in 2020 IEEE 92nd Vehicular Technology Conference (VTC2020-Fall), Nov 2020, pp. 1-5.

[17] M. Aeberhard and N. Kaempchen, "High-level sensor data fusion architecture for vehicle surround environment perception," in Proc. 8th Int. Workshop Intell. Transp, vol. 665, 2011.

[18] M. Gabb, H. Digel, T. Müller, and R. Henn, "Infrastructure-supported perception and track-level fusion using edge computing," in 2019 IEEE Intelligent Vehicles Symposium (IV), June 2019, pp. 1739-1745.

[19] M. Tsukada, T. Oi, M. Kitazawa, and H. Esaki, "Networked roadside perception units for autonomous driving," MDPI Sensors, vol. 20, no. 18, 2020.

[20] R. Azimi, G. Bhatia, R. R. Rajkumar, and P. Mudalige, "Stip: Spatiotemporal intersection protocols for autonomous vehicles," in 2014 ACM/IEEE International Conference on Cyber-Physical Systems (ICCPS), 2014, pp. 1-12.

[21] S. Aoki and R. Rajkumar, "Dynamic intersections and self-driving vehicles," in 2018 ACM/IEEE 9th International Conference on CyberPhysical Systems (ICCPS), 2018, pp. 320-330.

[22] K. Dresner and P. Stone, "A multiagent approach to autonomous intersection management," Journal of artificial intelligence research, vol. 31, pp. 591-656, 2008.

[23] B. Liu, Q. Shi, Z. Song, and A. El Kamel, "Trajectory planning for autonomous intersection management of connected vehicles," Simulation Modelling Practice and Theory, vol. 90, pp. 16 - 30, 2019.

[24] M. Bashiri and C. H. Fleming, "A platoon-based intersection management system for autonomous vehicles," in 2017 IEEE Intelligent Vehicles Symposium (IV), 2017, pp. 667-672.

[25] C. Englund, L. Chen, J. Ploeg, E. Semsar-Kazerooni, A. Voronov, H. H. Bengtsson, and J. Didoff, "The grand cooperative driving challenge 2016: boosting the introduction of cooperative automated vehicles," IEEE Wireless Communications, vol. 23, no. 4, pp. 146-152, 2016.

[26] O. Sawade, M. Schulze, and I. Radusch, "Robust communication for cooperative driving maneuvers," IEEE Intell. Transp. Syst. Mag., vol. 10, no. 3, pp. 159-169, 2018.

[27] V. Milanes, J. Godoy, J. Villagra, and J. Perez, "Automated On-Ramp merging system for congested traffic situations," IEEE Trans. Intell. Transp. Syst., vol. 12, no. 2, pp. 500-508, Jun. 2011.

[28] L. Eiermann, O. Sawade, S. Bunk, G. Breuel, and I. Radusch, "Cooperative automated lane merge with role-based negotiation," 2020 IEEE Intelligent Vehicles Symposium (IV), Oct. 2020.

[29] D. Heb, R. Lattarulo, J. Perez, J. Schindler, T. Hesse, and F. Koster, "Fast maneuver planning for cooperative automated vehicles," 201821 st
International Conference on Intelligent Transportation Systems (ITSC), 2018.

[30] M. Nichting, D. Hess, J. Schindler, T. Hesse, and F. Koster, "Space time reservation procedure (STRP) for V2X-Based maneuver coordination of cooperative automated vehicles in diverse conflict scenarios," 2020 IEEE Intelligent Vehicles Symposium (IV), Oct. 2020.

[31] J. Schindler, R. Markowski, D. Heß, D. Wesemeyer, C. Böker, B. Coll Perales, G. Thandavarayan, M. Sepulcre, J. Gozalvez, M. Rondinone, D. Matheis, and X. Zhang, "TransAID deliverable 7.2: System prototype demonstration (iteration 2)," Feb. 2021.

[32] A. Correa, R. Alms, J. Gozalvez, M. Sepulcre, M. Rondinone, R. Blokpoel, L. Lücken, and G. Thandavarayan, "Infrastructure support for cooperative maneuvers in connected and automated driving," in 2019 IEEE Intelligent Vehicles Symposium (IV). IEEE, 2019, pp. 20-25.

[33] A. Correa, F. Andert, R. Blokpoel, N. Wojke, G. Thandavarayan, B. Coll Perales, M. Sepulcre, J. Gozalvez, A. Wijbenga, J. Schindler, S. Maerivoet, X. Zhang, A. Leich, and C. Böker, "TransAID deliverable 5.2: V2X-based cooperative sensing and driving in transition areas (second iteration)," Mar. 2020.

[34] R. Jacob, M. Gay, M. Dod, S. Lorenz, A. Jungmann, L. Franke, M. Philipp, M. Kloeppel-Gersdorf, M. Haberjahn, E. Gruschka, and G. Fettweis, "IVS-KOM: A reference platform for heterogeneous ITS communications," in 2020 IEEE 92th Vehicular Technology Conference (VTC2020-Fall), 2020.

[35] R. Auerswald, R. Busse, M. Dod, R. Fritzsche, A. Jungmann, M. Klöppel-Gersdorf, J. F. Krems, S. Lorenz, F. Schmalfuß, S. Springer et al., "Cooperative driving in mixed traffic with heterogeneous communications and cloud infrastructure." in VEHITS, 2019, pp. 95-105.

[36] B. Hafner, J. Jiru, K. Roscher, J. Ott, G. A. Schmitt, and Y. Sevilmis, "CVIP: A protocol for complex interactions among connected vehicles," 2020 IEEE Intelligent Vehicles Symposium (IV), Oct. 2020.

[37] C. W. Daniele Bellan, "Decentralized cooperative collision avoidance for automated vehicles: a real-world implementation," 2020 IEEE Intelligent Vehicles Symposium (IV), Oct. 2020.

[38] M. Mizutani, M. Tsukada, and H. Esaki, "AutoMCM: Maneuver Coordination Service with Abstracted Functions for Autonomous Driving," in 24th IEEE International Conference on Intelligent Transportation (ITSC), Indianapolis, IN, United States, 2021.

[39] M. Quigley, K. Conley, B. Gerkey, J. Faust, T. Foote, J. Leibs, R. Wheeler, and A. Y. Ng, "Ros: an open-source robot operating system," in ICRA workshop on open source software, vol. 3, no. 3.2. Kobe, Japan, 2009, p. 5.

[40] T. Bray, "The JavaScript Object Notation (JSON) Data Interchange Format," RFC 7159 (Proposed Standard), Internet Engineering Task Force, Mar. 2014. [Online]. Available: http://www.ietf.org/rfc/rfc7159.txt

[41] ITS; Vehicular Communications; GeoNetworking; Part 4: Geographical addressing and forwarding for point-to-point and point-to-multipoint communications;, Std., Jul. 2014, ETSI EN 302 636-4-1 V1.2.1.

[42] A. d. S. S. da CML, "C-ROADS platform for C-ITS in europe," CROADS PORTUGAL STAKEHOLDER WORKSHOP, Nov. 2017. 